\title{
Importance of extended protease substrate recognition motifs in steering BNIP-2 cleavage by human and mouse granzymes $B$
}

Petra Van Damme $e^{1,2^{*}+}$, Kim Plasman ${ }^{1,2+}$, Giel Vandemoortele ${ }^{1,2}$, Veronique Jonckheere ${ }^{1,2}$, Sebastian Maurer-Stroh ${ }^{3,4}$ and Kris Gevaert ${ }^{1,2}$

\begin{abstract}
Background: Previous screening of the substrate repertoires and substrate specificity profiles of granzymes resulted in long substrate lists highly likely containing bystander substrates. Here, a recently developed degradomics technology that allows distinguishing efficiently from less efficiently cleaved substrates was applied to study the degradome of mouse granzyme B (mGrB).

Results: In vitro kinetic degradome analysis resulted in the identification of $37 \mathrm{mGrB}$ cleavage events, 9 of which could be assigned as efficiently targeted ones. Previously, cleavage at the IEAD 75 tetrapeptide motif of Bid was shown to be efficiently and exclusively targeted by human granzyme B ( $h G r B)$ and thus not by $\mathrm{mGrB}$. Strikingly, and despite holding an identical P4-P1 human Bid (hBid) cleavage motif, mGrB was shown to efficiently cleave the BCL2/adenovirus E1B $19 \mathrm{kDa}$ protein-interacting protein 2 or BNIP-2 at IEAD 28 . Like Bid, BNIP-2 represents a pro-apoptotic $\mathrm{BCl}-2$ protein family member and a potential regulator of $\mathrm{GrB}$ induced cell death. Next, in vitro analyses demonstrated the increased efficiency of human and mouse BNIP-2 cleavage by $\mathrm{mGrB}$ as compared to hGrB indicative for differing Bid/BNIP-2 substrate traits beyond the P4-P1 IEAD cleavage motif influencing cleavage efficiency. Murinisation of differential primed site residues in hBNIP-2 revealed that, although all contributing, a single mutation at the P3' position was found to significantly increase the $\mathrm{mGrB} / \mathrm{hGrB}$ cleavage ratio, whereas mutating the P1' position from $\mathrm{I}_{29}>\mathrm{T}$ yielded a 4-fold increase in $\mathrm{mGrB}$ cleavage efficiency. Finally, mutagenesis analyses revealed the composite BNIP-2 precursor patterns to be the result of alternative translation initiation at near-cognate start sites within the $5^{\prime}$ leader sequence (5'UTR) of BNIP-2.
\end{abstract}

Conclusions: Despite their high sequence similarity, and previously explained by their distinct tetrapeptide specificities observed, the substrate repertoires of mouse and human granzymes B only partially overlap. Here, we show that the substrate sequence context beyond the P4-P1 positions can influence orthologous granzyme B cleavage efficiencies to an unmatched extent. More specifically, in BNIP-2, the identical and $\mathrm{hGrB}$ optimal IEAD tetrapeptide substrate motif is targeted highly efficiently by $\mathrm{mGr}$, while this tetrapeptide motif is refractory towards $\mathrm{mGrB}$ cleavage in Bid.

Keywords: BNIP-2, Bid, Granzyme B, Extended substrate specificity, N-terminal COFRADIC, Near-cognate translation initiation, Degradomics

\footnotetext{
* Correspondence: Petra.VanDamme@vib-UGent.be

${ }^{\dagger}$ Equal contributors

${ }^{1}$ Department of Medical Protein Research, VIB, Flanders Interuniversity

Institute for Biotechnology, Ghent University, A. Baertsoenkaai 3, B9000

Ghent, Belgium

${ }^{2}$ Department of Biochemistry, Ghent University, B-9000 Ghent, Belgium

Full list of author information is available at the end of the article
}

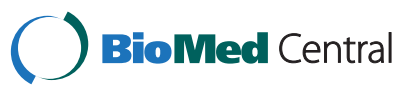

(c) 2014 Van Damme et al.; licensee BioMed Central Ltd. This is an Open Access article distributed under the terms of the Creative Commons Attribution License (http://creativecommons.org/licenses/by/4.0), which permits unrestricted use, distribution, and reproduction in any medium, provided the original work is properly credited. The Creative Commons Public Domain Dedication waiver (http://creativecommons.org/publicdomain/zero/1.0/) applies to the data made available in this article, unless otherwise stated. 


\section{Background}

While gene orthology implies homology between two genes separated by a speciation event, no conclusions regarding their full functional equivalence can be simply extrapolated. Contradictions in literature on apparent isofunctional orthologs are thus partly due to the fact that incorrect functional extrapolations between orthologous genes were made. Human granzyme B (hGrB), a protease belonging to a family of serine proteases present in the granules of cytotoxic lymphocytes (cytotoxic $\mathrm{T}$ lymphocytes and natural killer cells) and implicated in the induction of cell death, was shown to display distinct functional and structural characteristics as compared to its murine ortholog despite their $80 \%$ amino acid similarity and $70 \%$ amino acid identity [1-4]. Positional scanning synthetic combinatorial libraries, phage display data and proteomewide degradome analyses all aided in the elucidation of the differential specificity profiles and substrate repertoires of these orthologous granzymes [1-5]. Next to the higher cytotoxicity of hGrB, differences in cleavage efficiencies of Bid and caspase-3, substrates known to be of critical importance for the execution of $\mathrm{hGrB}$ induced cell death, were found for the human and mouse orthologous granzymes B [2-4]. While hGrB is capable of cleaving Bid and caspase- 3 orthologs with comparable cleavage efficiencies, $\mathrm{mGrB}$ is unable to process Bid and cleaves mouse caspase-3 about 12-fold more efficient as compared to human caspase-3 [4]. Differential positional proteomics analyses furthermore reported on a proteome-wide analysis of the extended specificity profiles (i.e., beyond cleavage positions P4-P1) of both orthologous granzymes, and showed enhanced $\mathrm{mGrB}$ versus hGrB cleavage efficiencies of substrates holding Lys and Gly residues in the P1' and $\mathrm{P} 2$ ' positions respectively (Pn-...-P2-P1-P1' -P2'...$--\mathrm{P} n$ ' nomenclature according to Schechter and Berger [6]) amongst other (subtle) distinct substrate traits [1].

The ability to separate critical from bystander cleavage events may also aid in elucidating the different underlying mechanisms of action engaged by these orthologous granzymes when exerting their primary functions [7-9]. Despite hindering a direct extrapolation to the physiological importance of $\mathrm{mGrB}$-induced cleavages, distinctive orthologous granzyme $B$ substrate traits can be captured using identical proteome backgrounds. As such, and in line with a previously developed positional proteomics strategy to follow substrate cleavage kinetics [8], we here made use of a human proteome background for identifying $\mathrm{mGrB}$ cleavage events. In this pioneering study on hGrB, of the $101 \mathrm{hGrB}$ cleavage sites identified, only 18 were found to be efficiently cleaved, including cleavage at $\mathrm{IEAD}_{75}$ in the BH3-interacting domain death agonist Bid [Swiss-Prot: P55957] and at $\mathrm{IEAD}_{28}$ in the pro-apoptotic Bcl-2 family member BNIP-2 [Swiss-Prot: Q12982] [10,11]. While Bid stands central in hGrB induced apoptosis [12], both human and mouse Bid were found to be very poor $\mathrm{mGrB}$ substrates [1-4]. The essential requirement of Bid cleavage for hGrB-induced cell death [13] and the Bid independent cytotoxicity of $\mathrm{mGrB}$ [14], led to the postulation that $\mathrm{mGrB}$ exerts its cytotoxicity via direct activation of an effector caspase (i.e., caspase 3 and/or 7) [2], by relieving IAP inhibition of caspase-3, as shown for hGrB [15], and/or by the direct cleavage of yet unidentified substrates.

To explore these hypotheses, we applied our degradomics technology to probe for efficient $\mathrm{mGrB}$ cleavage events. Next to caspase-7, and in analogy with a previous analysis probing for efficient hGrB cleavage events [8], BNIP-2 cleavage was here assigned amongst the most efficiently cleaved $\mathrm{mGrB}$ substrates. Members of the family of pro-apoptotic Bcl-2 and adenovirus E1B $19 \mathrm{kDa}$ interacting proteins to which BNIP-2 belongs, are known to interact with various pathological and physiological anti-apoptotic proteins such as Bcl-2 and the adenoviral E1B $19 \mathrm{kDa}$ protein [16]. In addition to several other domains, BNIP-2 holds a BNIP-2 and Cdc42GAP homology $(\mathrm{BCH})$ domain (Figure 1), via which it is able to bind cdc42, a member of the Rho subfamily involved in controlling cell morphological changes, to promote the formation of cellular extensions [17] and myogenic differentiation via yet unidentified regulator(s) [18].

Besides its implication in cellular differentiation, other functions have been attributed to BNIP-2 [19], including its involvement in apoptotic signaling (for human as well as mouse BNIP-2). Massive cell death of neuroblastoma cells occurs following overexpression of hBNIP-2, and is counteracted by treatment with neuroprotective estrogen, which results in a downregulation of hBNIP-2 [20]. These findings inspired several groups to study the molecular mechanisms underlying hBNIP-2 induced apoptosis. Interestingly, in vitro and/or in cellulo cleavage of hBNIP2 by the caspases $3,6,8,9$ and 10 occurs outside the $\mathrm{BCH}$ domain [21,22]. In addition, $\mathrm{GrB}$ mediated cleavage of hBNIP-2 could be observed when probing for human and mouse granzyme B substrates [1], a hGrB event furthermore predicted by a database search making use of the optimal Bid cleavage motif (i.e., IEAD) [11]. Human BNIP-2 was found to be directly processed at IEAD 28 by granzyme $\mathrm{B}$ in vitro and during natural killer cellmediated killing of tumor cells and thus not secondary to granzyme B induced caspase activation [11]. Although these data could suggest that caspase/granzyme-mediated release of the $\mathrm{BCH}$ domain influences the pro-apoptotic activity of BNIP-2, in line with the observed massive neuroblastoma cell death [20], full length hBNIP-2 by itself was able to induce cell death in HeLa cells [11].

Various signaling pathways, including activation of the intrinsic apoptotic pathway [23] or direct caspase activation [24], have been proposed to explain the apoptotic potential of BNIP-2. 


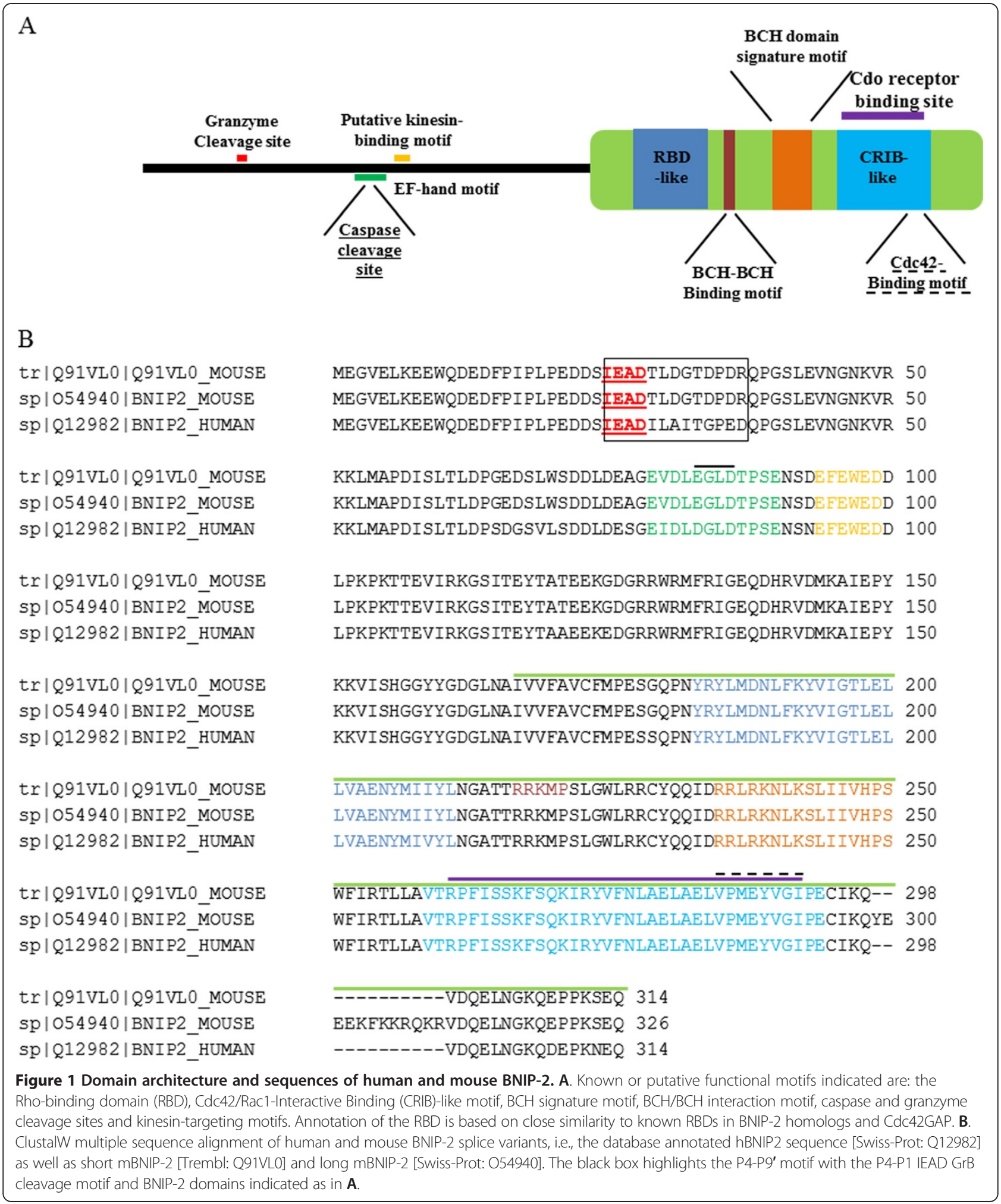

Overall, we here investigated the cleavage susceptibilities of BNIP-2 - identified in this study as an efficient mGrB substrate - by the orthologous mouse and human granzymes B and demonstrated that differential substrate targeting of BNIP-2 by orthologous granzymes B is strongly influenced by the primed site residues. 


\section{Methods}

\section{Ethics statement}

All results of this research were based on the use of cultured human (HeLa or Jurkat) or mouse (NIH/3T3 or YAC-1) cell lines. Neither human (human subjects or human derived material) nor animals (vertebrates or any regulated invertebrates) were used in this experimental research.

\section{Cell culture}

Human HeLa cells (ATCC CCL-2, American Type Culture Collection, Manassas, VA, USA), mouse NIH/3T3 cells (ATCC CRL-1658), human Jurkat cells (ATCC TIB-152) and mouse YAC-1 cells (ATCC; TIB-160) were grown in DMEM (HeLa and NIH/3T3) or RPMI-1640 (Jurkat and YAC-1) medium, supplemented with $10 \%$ foetal bovine serum (Invitrogen, Carlsbad, CA, USA), 100 units/ml penicillin (Invitrogen) and $100 \mu \mathrm{g} / \mathrm{ml}$ streptomycin (Invitrogen). For proteome analyses, Jurkat cells were grown in RPMI 1640 medium (Invitrogen, Carlsbad, CA, USA, \#61870-010) containing either natural, ${ }^{13} \mathrm{C}_{6}$ or ${ }^{13} \mathrm{C}_{6}^{15} \mathrm{~N}_{4}$ L-arginine (Cambridge Isotope Labs, Andover, MA, USA) at a concentration of $230 \mu \mathrm{M}$ (i.e., 20\% of its suggested concentration in RPMI at which L-arginine to proline conversion was not detectable). Media were supplemented with $10 \%$ dialyzed foetal bovine serum (Invitrogen, 26400-044), 100 units/ml penicillin (Invitrogen) and $100 \mu \mathrm{g} / \mathrm{ml}$ streptomycin (Invitrogen). Cells were cultured at $37^{\circ} \mathrm{C}$ and in $5 \% \mathrm{CO}_{2}$ for at least six population doublings to ensure complete incorporation of the labeled arginine.

\section{Preparation of cell lysates for N-terminal COFRADIC analysis}

SILAC-labeled Jurkat cells were pre-incubated with the pan-caspase inhibitor N-benzyloxycarbonyl-Val-Ala-Aspfluoromethyl ketone (z-VAD-fmk) at a final concentration of $50 \mu \mathrm{M}$ for $15 \mathrm{~min}$ at $37^{\circ} \mathrm{C}$, washed in D-PBS and re-suspended at $7 \times 10^{6}$ cells per $\mathrm{ml}$ in $50 \mathrm{mM}$ Tris- $\mathrm{HCl}$ $\mathrm{pH} 8.0$ and $100 \mathrm{mM} \mathrm{NaCl}$. Cells were lysed by three rounds of freeze-thawing. Supernatants, cleared by centrifugation for $10 \mathrm{~min}$ at 16,000 g, were treated with $200 \mathrm{nM}$ of mouse recombinant granzyme $B(m G r B)$ for the indicated time points at $37^{\circ} \mathrm{C}$. Recombinant $\mathrm{mGrB}$ was produced in Pichia pastoris as described previously [3]. Solid guanidinium hydrochloride was added to a final concentration of $4 \mathrm{M}$ in order to inactivate $\mathrm{mGrB}$ and denature all proteins. Before mixing equal amounts of samples, proteins were reduced and alkylated using TCEP.HCl (1 mM final concentration (f.c.)) and iodoacetamide ( $2 \mathrm{mM}$ f.c.) respectively, for $1 \mathrm{~h}$ at $30^{\circ} \mathrm{C}$. Subsequent steps of the $\mathrm{N}$-terminal COFRADIC protocol were performed as described previously [25]. The proteome was digested overnight at $37^{\circ} \mathrm{C}$ with sequencing-grade, modified trypsin (Promega, Madison, WI, USA) (enzyme/ substrate of $1 / 100 \mathrm{w} / \mathrm{w})$.

\section{LC-MS/MS analysis and data processing}

ESI LC-MS/MS analysis was performed on an ESI-Q-TOF Premier (Waters Corporation) mass spectrometer as described before [26]. ESI-Q-TOF MS/MS peptide fragmentation spectra were converted to pkl files using the Masslynx ${ }^{\ominus}$ software (version 4.1, Waters Corporation). $\mathrm{N}$-terminal peptides were identified using a locally installed version of the MASCOT database search engine version 2.2.1 and the Swiss-Prot database (version 13.0 of UniProtKB/Swiss-Prot protein database, containing in total 356,194 sequence entries from which 18,609 human entries) was searched with restriction to human proteins. The following search parameters were used. Peptide mass tolerance was set at $0.2 \mathrm{Da}$ and peptide fragment mass tolerance at $0.1 \mathrm{Da}$ with the ESI-QUAD-TOF as selected instrument for peptide fragmentation rules for the Q-TOF Premier data. Endoproteinase Arg-C/P (i.e., no restriction towards arginine-proline cleavage) was set as enzyme allowing one missed cleavage. Variable modifications were pyroglutamate formation of $\mathrm{N}$ terminal glutamine, pyrocarbamidomethyl formation of N-terminal alkylated cysteine, deamidation of asparagine, acetylation and tri-deuteroacetylation of the alpha-N-terminus. Fixed modifications were methionine oxidation (sulfoxide), carbamidomethylation of cysteine, tri-deuteroacetylation of lysine and, for identifying heavy labelled peptides, $\left[{ }^{13} \mathrm{C}_{6}\right]$ or $\left[{ }^{13} \mathrm{C}_{6}^{15} \mathrm{~N}_{4}\right]$-arginine were additionally set as fixed modifications. Only peptideto-spectrum matches with MASCOT ion scores that exceeded the corresponding MASCOT identity threshold score (at 95\% confidence level) and that were ranked one, were withheld. In addition, spectra that received a low MASCOT ion score (5 or less points above threshold for identity) were further interrogated and only spectra that contained b- and $\mathrm{y}$ - fragment ions covering a stretch of at least three consecutive amino acids were considered identified. The estimated false discovery rate by searching decoy databases was typically found to lie between 2 and $4 \%$ on the spectrum level [27]. Whenever a peptide matched to multiple members of a protein family (redundancy), the protein entry reported (column 'Protein description', Additional file 1: Table S1) was according to its alphabetical ranking. In each case, other matching protein entries were reported under the column 'Isoforms'. The peptide intensity ratios were manually calculated from the MS data in the Masslynx ${ }^{\odot}$ software version 4.1 environment. In order to correct for small variations in initial protein concentrations, the ${ }^{12} \mathrm{C}_{6} /{ }^{13} \mathrm{C}_{6}$ and ${ }^{12} \mathrm{C}_{6} /{ }^{13} \mathrm{C}_{6}^{15} \mathrm{~N}_{4}$ ratios of the 541 database-annotated protein $\mathrm{N}$-terminal peptides identified (peptides starting at position 1 or 2) were first subjected to robust statistics using the base- 2 logarithms 
of their ratios. The corresponding ratio means, -0.003 $\left({ }^{12} \mathrm{C}_{6} /{ }^{13} \mathrm{C}_{6}\right)$ and $0.009\left({ }^{12} \mathrm{C}_{6} /{ }^{13} \mathrm{C}_{6}^{15} \mathrm{~N}_{4}\right)$, were used to correct for the calculated ratios of all peptides identified. $\mathrm{R}$ was used to calculate the probability distributions of $\log 2$ transformed N-terminal peptide ratios between the 1st and 2 nd time point $(N(-0.003,0.1451)$ (mean, standard deviation)) and between the 2nd and 3rd time point $(N(0.009,0.1871))$. Protease substrate intensity ratios falling within the $98 \%$ probability interval of the protein N-terminal peptide intensity ratios were considered as derived from efficiently cleaved sites (see Results section and [8]). The mass spectrometry proteomics data have been deposited to the ProteomeXchange Consortium (http://proteomecentral.proteomexchange.org) via the PRIDE partner repository [28] with the dataset identifier PXD000204 and DOI 10.6019/PXD000204.

\section{Immunoblot analysis}

PBS washed Jurkat cells were re-suspended in $50 \mathrm{mM}$ Tris. $\mathrm{HCl} \mathrm{pH} 8.0$ and $100 \mathrm{mM} \mathrm{NaCl}$ and subjected to three rounds of freeze thaw lysis. Supernatants, cleared by centrifugation for $10 \mathrm{~min}$ at $16,000 \mathrm{~g}$, were pre-treated with $5 \mathrm{mM}$ iodoacetamide for $15 \mathrm{~min}$ in the dark to avoid downstream granzyme B mediated caspase activation [29]. Protein samples were subsequently treated with varying concentrations of hGrB or mGrB (ranging from $0.97 \mathrm{nM}$ to $1 \mu \mathrm{M}$ ). Following GrB incubation, sample loading buffer was added and equal amounts of protein ( $50 \mu \mathrm{g} / \mathrm{sample}$; protein concentrations were measured using the DC Protein Assay Kit from Bio-Rad (Munich, Germany)) were separated on $4-12 \%$ or $12 \%$ polyacrylamide Criterion XT-gels in MOPS buffer (Bio-Rad) at 150 V. Subsequently, proteins were transferred onto a PVDF membrane. To detect endogenous Bid and BNIP-2 proteins, membranes were blocked for $30 \mathrm{~min}$ in $5 \%$ skimmed milk powder containing Tris-buffered saline and $0.1 \%$ Tween20 (TBS-T) and probed for $1 \mathrm{~h}$ with primary antibody in $5 \%$ skimmed milk powder in TBS-T. Following three 10 min washes in TBS-T, membranes were incubated with species-specific HRP-conjugated antibody for $1 \mathrm{~h}$ in $5 \%$ skimmed milk powder containing TBS-T. Again following a 3-fold wash step in TBS-T or TBS (last wash step), bands were visualized on ECL Hyperfilms (Amersham Biosciences, Buckinghamshire, UK) using an enhanced chemiluminiscence kit (PerkinElmer Life Sciences, Boston, MA, USA). Bid antibody was from R\&D systems (AF860) and BNIP-2 antibody from Hypromatrix (HM1251).

\section{Assessing BNIP-2 peptide substrate cleavage}

An oligopeptide holding the hBNIP-2 GrB cleavage site (italics) identified using N-terminal COFRADIC, $\mathrm{NH}_{2}$.LPEDDSIEADILAITGY $\left(\mathrm{NO}_{2}\right)$ R.OH, was synthesized in-house using Fmoc-chemistry on an Applied Biosystems 433A Peptide Synthesizer. Cleavage progression was assayed for $2 \mathrm{~h}$ at $37^{\circ} \mathrm{C}$ with various concentrations of human and mouse $\mathrm{GrB}$ (50, 200 and $500 \mathrm{nM}$ ) at a substrate concentration of $100 \mu \mathrm{M}$. The reactions were stopped by adding trifluoroacetic acid to a final concentration of $1 \%$. Cleavage by human and mouse GrB was assessed by monitoring the UV absorbance signal $(214 \mathrm{~nm})$ of the precursor peptide and its fragments upon reverse-phase HPLC separation. The extent of hydrolysis was determined from the intensity of the product peak(s) compared to the intensity of their unprocessed precursor.

\section{Granzyme cleavage of in vitro transcribed and translated BNIP-2}

Vectors encoding wild type BNIP-2 (with or without 5' leader) and BNIP-2 variants were used as templates for in vitro coupled transcription/translation in a rabbit reticulocyte lysate system according to the manufacturer's instructions (IVTT; Promega) to generate $\left[{ }^{35} \mathrm{~S}\right]$ methionine-labeled BNIP-2 (variants). The TnT Quick Master Mix (40 $\mu$ l) (pOTB7-hBNIP-2 (RZPD Imagenes, cat $n^{\circ}$ IRAUp969D059D, Germany) and pCMV-SPORT6mBNIP-2 (RZPD Imagenes, cat $n^{\circ}$ IRAVp968F0522D, Germany)) - consisting of a rabbit reticulocyte lysate, reaction buffer, SP6 or T7 RNA polymerase, an amino acid mixture without methionine and RNasin Ribonuclease Inhibitor - was mixed with $2 \mu \mathrm{l}\left[{ }^{35} \mathrm{~S}\right]$-methionine $(20 \mu \mathrm{Ci} / \mathrm{ml}), 1 \mu \mathrm{g}$ of plasmid DNA template $(1 \mu \mathrm{g} / \mu \mathrm{l})$ and $7 \mu \mathrm{l}$ nuclease-free water. Following $90 \mathrm{~min}$ incubation at $30^{\circ} \mathrm{C}$, translates were incubated with iodoacetamide (f.c. $5 \mathrm{mM}$ ) for $15 \mathrm{~min}$ at $37^{\circ} \mathrm{C}$ in the dark to avoid downstream caspase activation upon incubation with granzyme $B$ [30]. The translates $(5 \mu l)$ were subsequently incubated for $1.5 \mathrm{~h}$ at $37^{\circ} \mathrm{C}$ in granzyme assay buffer with varying concentrations of $\mathrm{hGrB}$ or $\mathrm{mGrB}$ (ranging from $0.97 \mathrm{nM}$ to $1 \mu \mathrm{M})$ in a total volume of $30 \mu \mathrm{l}$. Cleavage reactions were stopped by the addition of NuPAGE ${ }^{\bullet}$ LDS Sample Buffer (Invitrogen) and by heating the samples for $10 \mathrm{~min}$ at $70^{\circ} \mathrm{C}$. Proteins were separated on $4-12 \% \mathrm{NuPAGE}^{\circ}$ Bis-Tris gradient gels and subsequently transferred onto a PVDF membrane as indicated above, air-dried and exposed to a film suitable for radiographic detection (ECL Hyperfilms, Amersham Biosciences, Buckinghamshire, UK). The percentage of cleavage was determined by densitometry following autoradiographic analysis. Quantification was performed using the ChemiGenius imaging system (SynGene, Cambridge, United Kingdom) and signal intensity was quantified using GeneTools 3.07 image analysis software (SynGene).

\section{Cloning of wild type BNIP-2 and BNIP-2 variants}

Of note, the UniProtKB database contains entries of two mouse BNIP-2 splice variants (Swiss-Prot O54940 (long mouse BNIP-2) and Trembl Q91VL0 (short mouse 
BNIP-2)) which only differ by 12 additional amino acid residues at the $\mathrm{C}$-terminus (Figure $1 \mathrm{~B}$ ). All experiments conducted however (unless indicated otherwise) made use of the short mBNIP-2 variant as this represents the closest hBNIP-2 ortholog (Figure 1B). The pOTB7-hBNIP-2, the pCMV-SPORT6-mBNIP-2 (short mouse BNIP-2) and the pFLCI-mBNIP-2 (long mouse BNIP-2; RZPD Imagenes, cat $n^{\circ}$ E330010L14) vectors served as templates to generate attB-flanked PCR products of full-length and mutated BNIP-2 variants suitable for use in a Gateway ${ }^{\circ}$ BP recombination reaction with a donor vector (pDONR221, Invitrogen, cat $\left.n^{\circ} 12536-017\right)$ thereby creating an entry clone. Forward primers of human, short mouse and long mouse BNIP-2 (with or without 5 ' leader) and their respective reverse primers used to generate attB flanked PCR products are indicated in Additional file 2: Table S2. Of note, short and long mBNIP-2 reverse primers are identical and therefore annotated as mBNIP-2 reverse primer. Additional forward primers were designed to mimic the $\mathrm{GrB}$ cleaved C-terminal human and mouse BNIP-2 fragment (i.e., truncated h/m BNIP-2 or tBNIP-2), however additionally encoding an extra N-terminal Met residue in order for translation (initiation) to proceed, thereby giving rise to a tBNIP-2 with a Met-Ile- starting protein $\mathrm{N}$-terminus where Ile represents the $\mathrm{P} 1{ }^{\prime} \mathrm{Ile}_{29}$ of $\mathrm{GrB}$ cleaved full-length BNIP2. The reverse primers were designed to fuse the desired PCR products in frame with the C-terminal V5/His-tag encoded by the destination vector. To create BNIP-2 expression clones, the BNIP-2 inserts of the entry vectors were recombined into the pEF-DEST51 destination vector (Invitrogen, cat $n^{\circ}$ 12285-011) using LR-clonase (Invitrogen, cat $n^{\circ} 11791-$ 020) according to the manufacturer's instructions.

\section{Site-directed PCR-mutagenesis}

Plasmids encoding the human to mouse specific mutations: $\mathrm{I}_{29}>\mathrm{T}, \mathrm{A}_{31}>\mathrm{D}, \mathrm{I}_{32}>\mathrm{G}, \mathrm{G}_{34}>\mathrm{D}, \mathrm{E}_{36}>\mathrm{D}$ and $\mathrm{D}_{37}>\mathrm{R}$ as well as combined mutants of hBNIP-2 were generated by site-directed PCR-mutagenesis (QuickChange, Stratagene) according to the manufacturer's instructions using primer pairs listed in Additional file 2: Table S2. To create the hBNIP-2 mutant in which $\mathrm{P}^{\prime}, \mathrm{P}^{\prime}$ and $\mathrm{P} 8$ ' are mutated, the hBNIP-2 P1' mutant was used as template and forward and reverse primer pairs were created to generate this combined mutant (Additional file 2: Table S2; combined 1). The hBNIP-2 mutant where all differential amino acids C-terminal to the cleavage site were mutated to the corresponding amino acids present in the murine BNIP-2 ortholog, also made use of the P1' mutant as template. In first instance, primer pairs were developed as to generate a combined $\mathrm{P} 1^{\prime}, \mathrm{P} 3{ }^{\prime}, \mathrm{P} 4{ }^{\prime}, \mathrm{P} 6{ }^{\prime}$ and $\mathrm{P} 8{ }^{\prime}$ mutant (Additional file 2: Table S2; combined 2) upon which an additional PCR reaction was conducted to mutate P9' (Additional file 2: Table S2; combined 3). In human and mouse (both short and long) BNIP-2 expression plasmids, next to mutation of the database annotated AUG translation sites, mutations in their $5^{\prime}$ leader sequence at the upstream non-cognate (non-AUG) translation initiation sites (uTIS) reported in $[31,32]$ were created to monitor BNIP-2 N-terminal isoform production (Additional file 2: Table S2). The correctness of all (mutant) cDNA sequences generated was confirmed by DNA sequencing.

\section{BNIP-2 expression}

Human HeLa and mouse NIH/3T3 cells seeded one day prior to transfection at $50 \times 10^{3}$ cells/well in a 12 -well plate, were transiently transfected for $24 \mathrm{~h}$ with 0.6 or $0.8 \mu \mathrm{g} / \mathrm{ml}$ (final concentration) of the eukaryotic expression vectors using Fugene HD (Roche; cat. n 04709705001) according to the manufacturer's instructions. After $28 \mathrm{~h}$, cells were lysed on-plate using $80 \mu \mathrm{l}$ lysis buffer $(50 \mathrm{mM}$ Tris- $\mathrm{HCl}$ (pH 7.8), $150 \mathrm{mM} \mathrm{NaCl}, 1 \% \mathrm{NP}-40$ and protease inhibitor cocktail tablet (Roche, cat $\left.\mathrm{n}^{\circ} 11697498001\right)$ ), subjected to three rounds of freeze-thaw lysis and centrifuged for $10 \mathrm{~min}$ at 16,000 g. Sample loading buffer was then added and proteins were separated using 4-12\% polyacrylamide Criterion XT-gels (1.0 mm thick gels) in MOPS buffer (Bio-Rad) at 150 V. Subsequently, proteins were transferred onto a PVDF membrane. Membranes were blocked for $30 \mathrm{~min}$ in a 1:1 Tris-buffered saline and $0.1 \%$ Tween-20 (TBS-T) Odyssey Blocking solution (LI-COR, cat $\mathrm{n}^{\circ}$ 927-40003) and probed for $1 \mathrm{~h}$ with primary anti-V5 antibody (Invitrogen, cat $n^{\circ}$ R960-25, dilution: 1/5000) in TBS-T/Odyssey blocking buffer. Following three $10 \mathrm{~min}$ washes in TBS-T, membranes were incubated with secondary anti-mouse antibody (IRDye $800 \mathrm{CW}$ goat anti-mouse antibody IgG, LI-COR, cat $\mathrm{n}^{\circ}$ 926-32210) for $1 \mathrm{~h}$ in TBS-T/Odyssey blocking buffer followed by 3 washes in TBS-T or TBS (last wash step), bands were visualized using an Odyssey infrared imaging system (LI-COR).

\section{SLO mediated delivery of GrB}

$24 \mathrm{~h}$ before streptolysin-O (SLO)/GrB incubation, HeLa and NIH/3T3 cells, plated one day before transfection at $50 \times 10^{3}$ cells/well in a 12 -well plate, were transfected with 0.6 and $0.8 \mu \mathrm{g}$ BNIP-2 as described above. After $24 \mathrm{~h}$, cells were washed twice with serum-free and antibiotics-free DMEM medium and pretreated with $50 \mu \mathrm{M} \mathrm{z}$-VAD-fmk for $15 \mathrm{~min}$ at $37^{\circ} \mathrm{C}$ to inhibit caspase activation. Subsequently, cells were incubated with a sublytic concentration of SLO $(250 \mathrm{ng} / \mathrm{ml}$ ) (as determined by flow cytometry; data not shown) and $200 \mathrm{nM}$ $\mathrm{GrB}$ for $30 \mathrm{~min}$ at $37^{\circ} \mathrm{C}$. After $30 \mathrm{~min}$, an equal volume of DMEM containing 20\% FCS (f.c. 10\%) without antibiotics was added to inhibit SLO and cells were allowed to recover for $1 \mathrm{~h}$. Next, cells were lysed in SDS-PAGE loading buffer (60 mM Tris $\mathrm{pH} 6.8,10 \%$ glycerol and 2\% 
SDS) and the lysates cleared by centrifugation for $10 \mathrm{~min}$ at $16,000 \mathrm{~g}$. Protein samples were further processed as described above for immunoblot analysis and BNIP-2 was visualized using an anti-V5 antibody (Invitrogen, cat $\mathrm{n}^{\circ}$ R960-25; 1/5000 dilution).

\section{Results}

Mouse granzyme B kinetic degradomics

Screening the degradome of $\mathrm{mGrB}$ in function of time allows classifying substrate cleavage events according to the efficiency by which they get cleaved [8]. Here, three freeze-thaw Jurkat cell lysates differentially SILAC (Stable Isotope Labeling by Amino acids in Cell culture [33]) labeled by either normal $\left({ }^{12} \mathrm{C}_{6}\right),{ }^{13} \mathrm{C}_{6}$ or ${ }^{13} \mathrm{C}_{6}^{15} \mathrm{~N}_{4}$ L-arginine were incubated with $200 \mathrm{nM} \mathrm{mGrB}$ for 10,30 and $60 \mathrm{~min}$ respectively, as this allows monitoring the dynamics of $\mathrm{mGrB}$ generated neo-N-termini. Overall, this analysis resulted in the identification of 2,312 MS/MS spectra linked to $800 \mathrm{~N}$-terminal peptides. Of these, 556 (or $70 \%$ ) represent database annotated protein $\mathrm{N}$-termini (start position 1 or 2 and N-terminally modified with a(n) (trideutero)acetyl group), while 199 N-termini (or 25\%), originating from 191 proteins hinted to proteolytic events, potentially generated by $\mathrm{mGrB}$, as these did not map to position 1 or 2 while carrying a trideuteroacetyl moiety at their N-terminus. The remaining $45 \mathrm{~N}$-termini (or 5\%) might point to alternative translation initiation events due to the presence of a co-translationally added $\mathrm{N}$-terminal acetyl moiety [34]. To assign mGrB-specific cleavage events, all neo-N-termini were compared to a previous compilation of $\mathrm{GrB}$ cleavage events, the latter additionally making use of an internal control setup (i.e., a setup where no $\mathrm{GrB}$ was added) [1]. As a result, $37 \mathrm{mGrB}$ cleavage sites in 36 human proteins could unequivocally be assigned.

In our setup, protein $\mathrm{N}$-termini are generally not expected to change over time and their corresponding precursor ion intensities in MS should remain constant. As such, mGrB generated neo-N-termini with similar fold changes as compared to protein-N-termini point to efficiently cleaved substrates as the concentration of such neo-N-termini does not change upon prolonged protease incubation [8]. Neo-N-termini with increasing ion intensities in function of time can be considered as less efficiently cleaved (i.e., the concentration of such neo$\mathrm{N}$-termini increases upon prolonged protease incubation). By taking the intra-experimental variation of the 541 database (UniProtKB) annotated protein-N-termini for which the ${ }^{13} \mathrm{C}_{6} /{ }^{12} \mathrm{C}_{6}$ and ${ }^{13} \mathrm{C}_{6}^{15} \mathrm{~N}_{4} /{ }^{13} \mathrm{C}_{6}$ ratios could be calculated, a $98 \%$ confidence interval could be applied to distinguish efficient from less efficient mGrB cleavage sites (Figure 2). As a result, 9 cleavage sites were assigned efficiently cleaved as compared to 28 less efficiently cleaved (bystander) sites (Additional file 1: Table S1). In line with the hypothesis that $\mathrm{mGrB}$ induced apoptosis mainly proceeds via the activation of caspases [2,14], procaspase 7 appears amongst the most efficiently cleaved $\mathrm{mGrB}$ substrates. As expected, and in contrast to hGrB, (efficient) Bid cleavage was not observed [2-4]. Interestingly, another proapoptotic Bcl2-family member, BNIP-2 was found amongst the most efficiently cleaved $\mathrm{mGrB}$ substrates, as was the case for hGrB induced BNIP-2 cleavage $[1,8]$. More strikingly and in sharp contrast to the exclusive hGrB mediated cleavage of hBid [4], hBNIP-2 was found to be cleaved by $\mathrm{mGrB}$ at the identical and hGrB specific P4-P1 hBid motif IEAD.

\section{Cleavage efficiency of BNIP-2 by GrB: Biochemical characterization of (murinized) BNIP-2 by oligopeptide and substrate cleavage}

Western blot analyses using either hGrB or mGrB incubated human and mouse cell-free extracts validated our proteomics findings (Figure 3 and [8]). Cleavage of mBNIP-2 in mouse YAC-1 lysates however was not observed since BNIP-2 protein expression could not be detected even when using several BNIP-2 antibodies targeting different epitopes (data not shown). Noteworthy, both endogenous and recombinant BNIP-2 expression patterns revealed the presence of multiple BNIP-2 variants, i.e., N-terminal extended BNIP-2 variants produced by alternative translation initiation at near-cognate start codons in the 5 ' leader sequence as shown below. Overall, whereas both orthologous granzymes efficiently target hBNIP-2, human and mouse Bid processing could only be observed for hGrB (Figure 3B and C). Since multiple proteolytic BNIP-2 fragments could be observed (Figure 3A) and in order to probe for $\mathrm{GrB}$ cleavage efficiencies at the $\mathrm{IEAD}_{28}$ cleavage motif identified by positional proteomics, peptides holding the hBNIP-2 P10-P6' cleavage motif were synthesized and challenged with human or mouse GrB. Peptide cleavage analyses revealed that, while holding a hGrB specific P4-P1 hBid motif, mGrB did cleave the hBNIP-2 peptide at $\mathrm{IEAD}_{28}$ (Figure 4). Taking into account the greater cytotoxicity potential of $\mathrm{hGrB}$ versus $\mathrm{mGrB}$, and the fact that so far only $16 \%$ of all cleavages identified in a proteome-wide degradomics screen were found to be uniquely or more efficiently cleaved by $\mathrm{mGrB}$, mGrB cleaves the hBNIP-2 peptide about 5 times more efficient as compared to hGrB [2,3] (Figure 4).

To assess the contribution of BNIP-2 primed site residues in steering hGrB and mGrB cleavage efficiencies, (P' mutants of) in vitro translated human [Swiss-Prot: Q12982] and mouse [Trembl: Q91VL0] BNIP-2 were incubated with varying concentrations of human or mouse granzyme B. In analogy to cleavage efficiencies observed when probing cell lysates, this analysis revealed efficient hBNIP-2 cleavage by hGrB and mGrB, however additionally demonstrated that mBNIP-2 was cleaved far less efficient by hGrB $( \pm$ a 25 -fold reduction based on the 


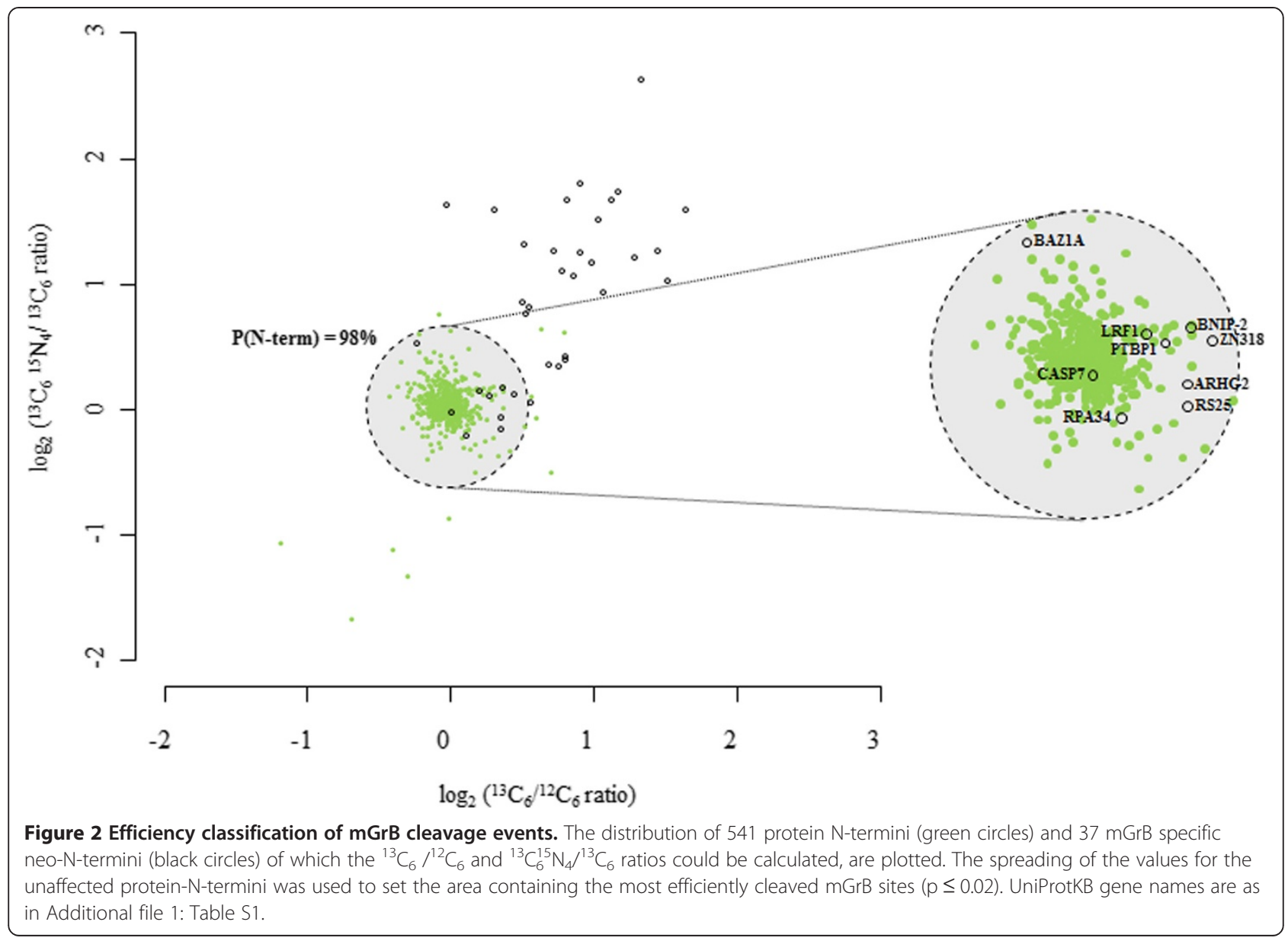

amount of protease needed to cleave $50 \%$ of the substrate), whereas both human and mouse BNIP-2 were cleaved with similar mGrB cleavage efficiencies (Figure 5). Taken together, this hints to the presence of (a) putative hGrB discriminating substrate feature(s). Interestingly, comparison of the human and mouse BNIP-2 protein sequences revealed a very high sequence similarity and identity of $96 \%$ and $93 \%$ respectively with the highest degree of divergence at the primed site sequence immediately following the scissile bond (i.e., from $\mathrm{P}^{\prime}$ to $\mathrm{P}^{\prime}$, next to 5 non-conservative substitutions, only 3 identical and 1 conservative amino acid substitution are found, Figure 1B).

We therefore investigated the contribution of each differing amino acid to $\mathrm{GrB}$ processing in the primed extended specificity profile of the $\mathrm{IEAD}_{28}$ motif. Using site-directed mutagenesis, we showed that the IEAA 28

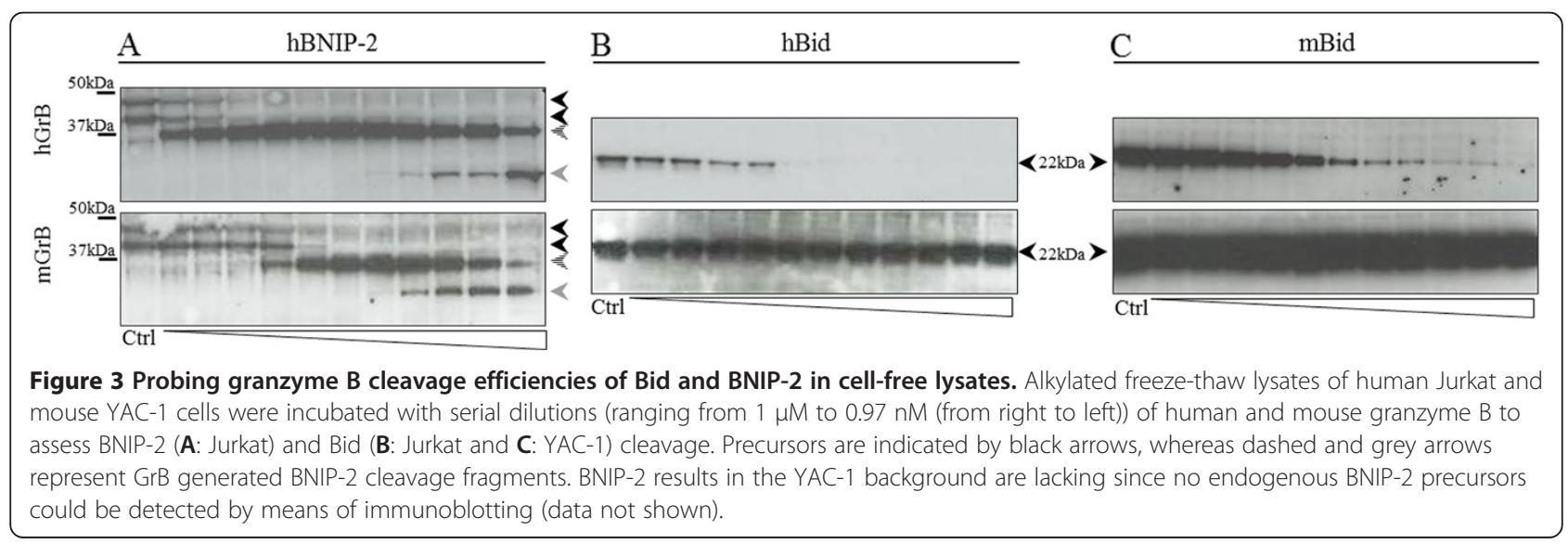




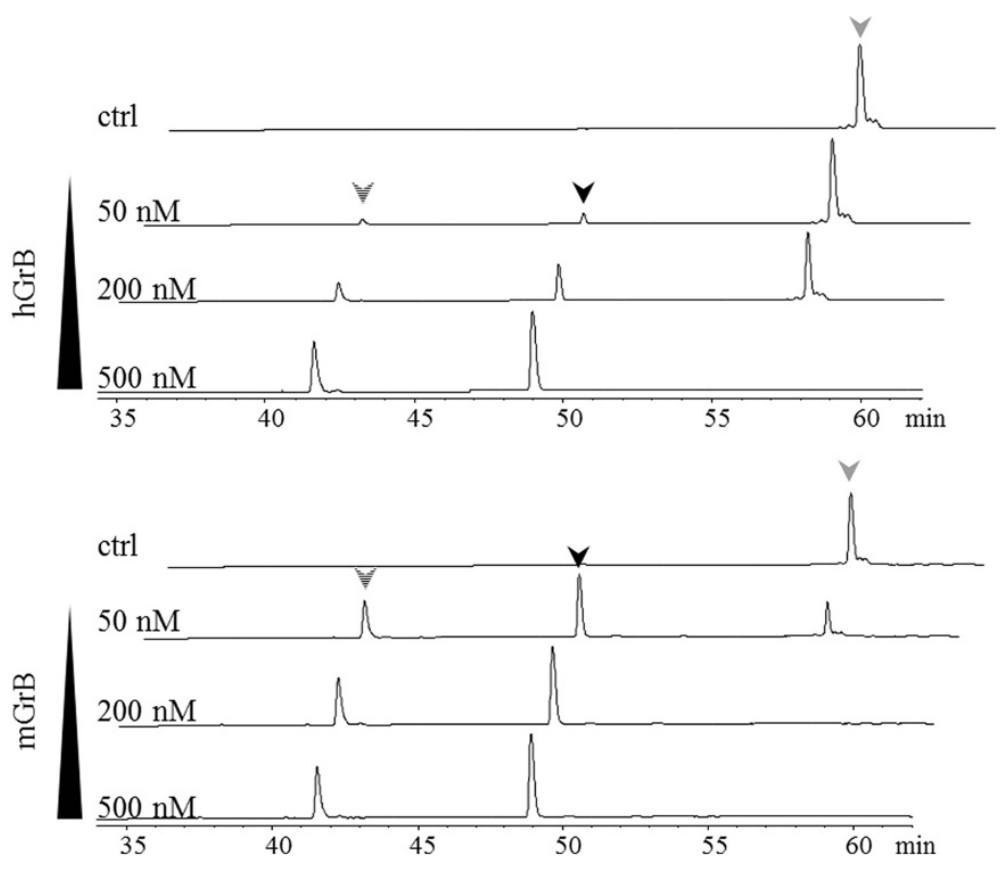

Figure 4 Granzyme B mediated cleavage of a peptide substrate holding the hBNIP-2 cleavage motif. Cleavage progression of the hBNIP-2 peptide substrate $\mathrm{NH}_{2}$.LPEDDSIEADILAITGY $\left(\mathrm{NO}_{2}\right) \mathrm{R}$.OH was assayed for $2 \mathrm{~h}$ at $37^{\circ} \mathrm{C}$ with various concentrations of human and mouse $\mathrm{GrB}(50,200$ and $500 \mathrm{nM}$ ) at a substrate concentration of $100 \mu \mathrm{M}$, and its precursor and fragment peptides separated by RP-HPLC (chromatograms with absorbance at $214 \mathrm{~nm}$ are shown). The grey arrow indicates (residual) precursor peptide, whereas black and dashed arrows indicate peptide fragments generated upon human (upper panel) and mouse (lower panel) GrB mediated cleavage at IEAD (the dashed arrow corresponds to the $\mathrm{N}$-terminal fragment $\mathrm{NH}_{2}$.LPEDDSIEAD.OH, whereas the black arrow corresponds to the C-terminal fragment $\mathrm{NH}_{2}$.ILAITGY(NO 2$)$ R.OH).

mutant was no longer processed by $\mathrm{hGrB}$ or $\mathrm{mGrB}$ (Additional file 3: Figure S1). Next, we "murinized" hBNIP-2 to investigate possible influences of the P1'-P9' primed site motif on GrB-mediated BNIP-2 proteolysis (Figure 6 and Additional file 4: Figure S2). These analyses reveal that, although all analyzed $\mathrm{P}^{\prime}$ positions contribute to the differences in $\mathrm{mGrB} / \mathrm{hGrB}$ cleavage efficiencies observed, the P3' $\mathrm{Ala}_{31}$ to Asp mutation increases the $\mathrm{mGrB} / \mathrm{hGrB}$ ratio of cleavage over 15 -fold, by and large explaining the poor cleavage susceptibility of mBNIP-2 by hGrB (Figure 6A). Furthermore, cleavage of this single amino acid mutant by $\mathrm{hGrB}$ was about 5 -fold reduced as compared to cleavage of wild-type hBNIP-2, whereas mGrB cleavage susceptibility was not affected. An Ile to Thr mutation at the $\mathrm{P} 1$ ' position yields the highest increase in cleavage efficiency for both human and mouse $\mathrm{GrB}$ as this variant was cleaved about 4-fold more efficient by both orthologous granzymes as compared to wild-type hBNIP-2 (Figure 6B). In addition, mutation of P6' and P8' amino acids to the corresponding mouse residues both appeared favorable for cleavage by both granzymes as compared to cleavage of wild-type hBNIP-2, whereas mutation of the P9' residue did not alter cleavage efficiency (Additional file 4: Figure S2). The creation of combined primed site mutants, such as the hBNIP-2 mutants containing the mBNIP-2 P1' $^{\prime}, \mathrm{P}^{\prime}$ and $\mathrm{P}^{\prime}$ residues, or all differentiating primed site amino acids (Figure 7), also increased the $\mathrm{mGrB} / \mathrm{hGrB}$ cleavage ratio over 10 -fold, indicative for the fact that multiple primed site residues contribute to the increased $\mathrm{mGrB} / \mathrm{hGrB}$ cleavage ratio as compared to hBNIP-2.

\section{BNIP-2 cleavage upon SLO-mediated GrB delivery in cellulo}

To assess if our in vitro findings can be extrapolated to cell cultures and to corroborate the results reported by Scott et al. [11] who demonstrated cleavage of hBNIP-2 by $\mathrm{hGrB}$ during natural killer mediated cell death, we pursued $\mathrm{GrB}$ cleavage of overexpressed human and mouse BNIP-2 by means of SLO-mediated GrB delivery in HeLa and NIH/3T3 cells respectively (Figure 8). Indeed, our results show that next to human BNIP-2, overexpressed mouse BNIP-2 is efficiently cleaved during human and mouse GrB induced cell death. Transfection of truncated BNIP-2 (i.e., the GrB generated cleavage fragment (tBNIP-2)) confirms cleavage at the IEAD $_{28}$ motif of full-length BNIP-2. However, whereas previous studies $[11,23,24]$ reporting on the overexpression of full-length and/or truncated hBNIP-2 led to moderate poly (ADP) ribose polymerase 1 (PARP-1) cleavage and/or caspase activation, indicative for apoptosis, when transfecting (truncated) human and mouse BNIP-2 variants, we were unable to observe caspase activity or any of the 


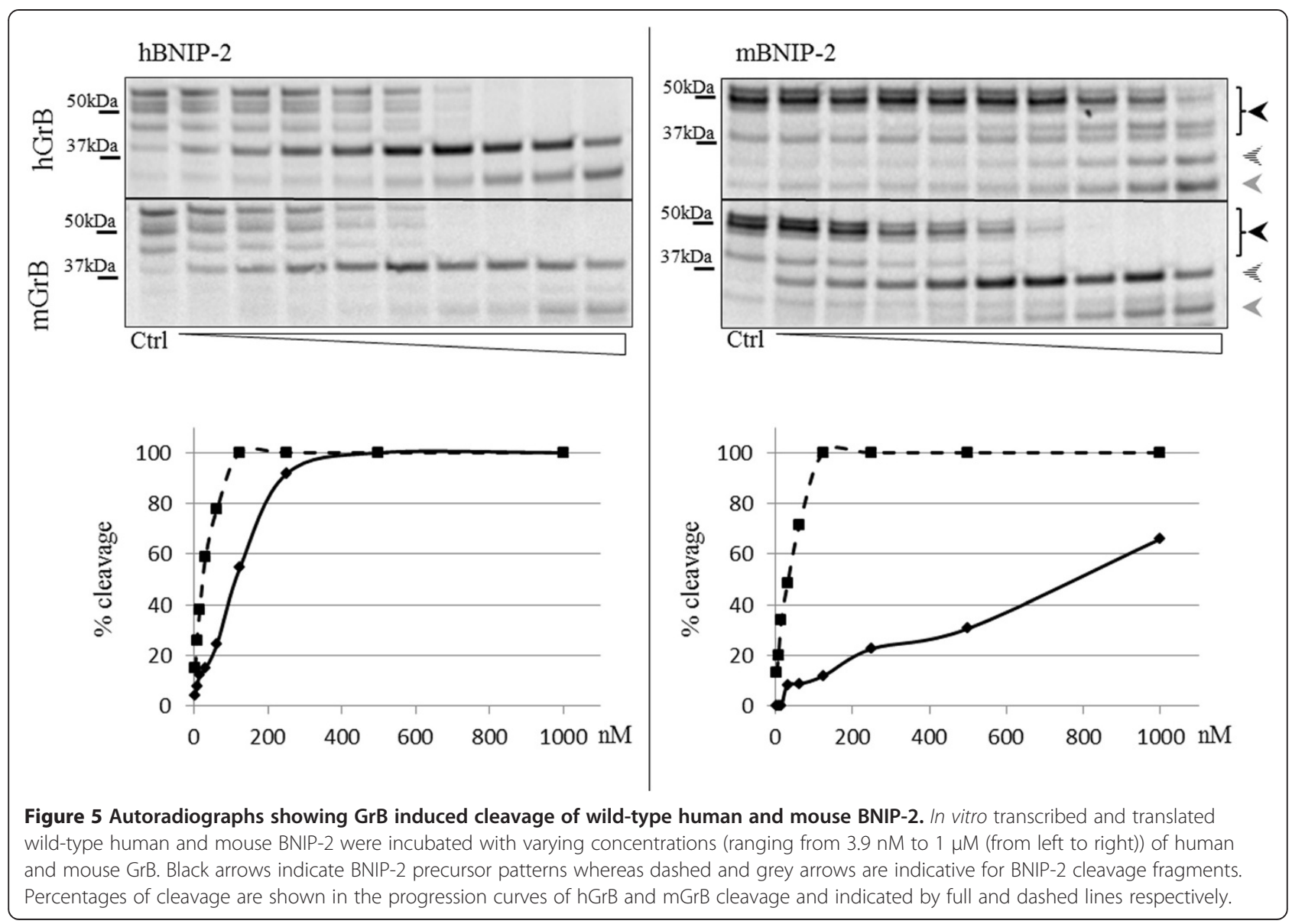

typical apoptosis related hallmarks using fluorimetric caspase assays and flow cytometry (data not shown).

\section{Assessing the influence of 5' leader sequences on BNIP-2 precursor patterns}

In line with previous findings in various human cell lines (i.e., NK and Daudi [11]) and when probing for endogenous or in vitro translated BNIP-2, in each case clearly distinguishable higher molecular BNIP-2 precursor bands could be observed (Figures 3, 5, 6, 7 and 8). Previously, these BNIP-2 variants were hypothesized to result from posttranslational modifications [35] or alternative splicing, as is the case for two other BNIP-2 homologs [36]. Of note also is the presence of two mBNIP-2 splice variants in the UniProtKB database (here referred to as short [Trembl: Q91VL0] and long mouse BNIP-2 [Swiss-Prot: O54940]), only differing in 12 additional amino acids close to the $\mathrm{C}$-terminus harbored in its $\mathrm{BCH}$ domain (Figures $1 \mathrm{~B}$ and 9A).

Genome-wide translation initiation profiling using RiboSeq $[31,32]$ however indicates that the higher molecular weight BNIP-2 species might represent N-terminally extended BNIP-2 variants raised by alternative translation initiation at near-cognate start codons in the $5^{\prime}$ leader sequence or presumed 5'UTR of BNIP-2. Ribosome profiling allows sequencing of ribosome-protected mRNA fragments [31] and, combined with the use of translation inhibitors that cause accumulation of ribosomes at initiation codons, the exact translation initiation sites and ORFs can be delineated [31,32]. Genome-wide analyses of in vivo translation in mouse embryonic stem cells and human Hek293t cells led to the identification of respectively one human and two mouse upstream in-frame translation initiation site(s) potentially giving rise to $\mathrm{N}$-terminally extended BNIP-2 variants (Figure 9A and Additional file 5 and Additional file 6: Figures S3 and S4). Since the original expression plasmids did hold part of the 5 ' leader sequence (53\% and $82 \%$ of the hBNIP-2 and mBNIP-2 5' leader sequences respectively) (Additional file 5 and Additional file 6: Figures S3 and S4), we wanted to experimentally monitor translation initiation at the nearcognate start codons upstream and in-frame of the database annotated translation initiation start site (dbTIS). Therefore, we created a mBNIP-2 expression plasmid deprived of its 5 ' leader sequence and examined the precursor profile upon transfection in human HeLa and mouse NIH/3T3 cells (Figure 9B). Indeed, the higher molecular weight precursor band could no longer be observed. 


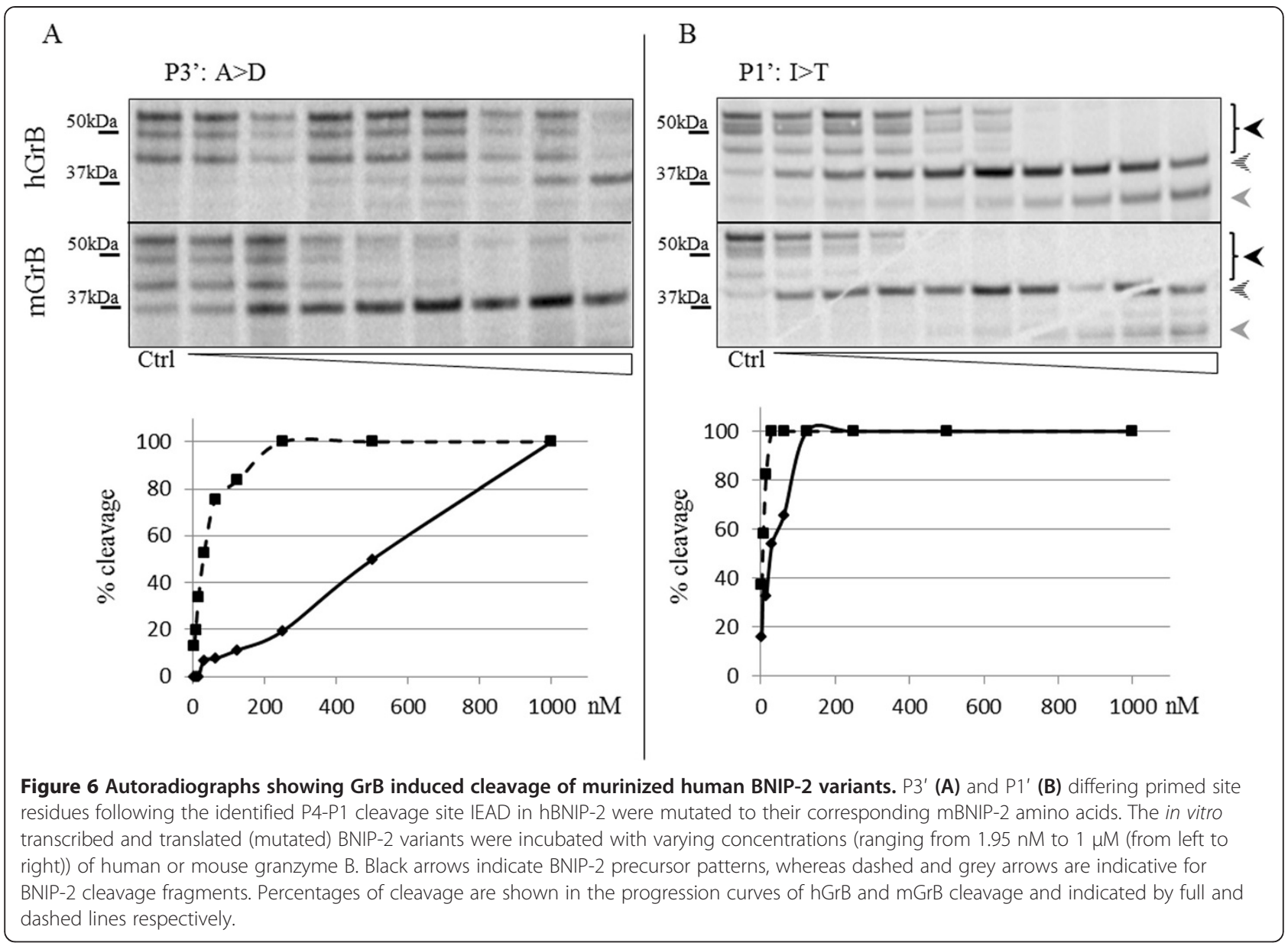

Given that translation initiation at upstream TIS (uTIS) A (a ctg codon in hBNIP-2 and atc in both mBNIP-2 variants) and uTIS B (tgg in hBNIP-2 and ctg in both mBNIP-2 variants) or uTIS C (ctg in all BNIP-2 variants studied) in theory increases the molecular weight of the database annotated BNIP-2 by $7 \mathrm{kDa}$ and $4.5 \mathrm{kDa}$ respectively (Figure 9A and Additional file 5 and Additional file 6: Figures S3 and S4), these findings correlate well with the different molecular weight precursor bands observed when probing endogenous, overexpressed and in vitro translated BNIP-2 (Figures 3, 5, 6, 7 and 8) [11]. To investigate this in more detail, BNIP-2 translation products were assessed in $\mathrm{HeLa}$ and NIH/3T3 cells transfected with human and mouse BNIP-2 expression plasmids mutated at each of the putative uTIS (uTIS A-C) reported (Figure 10). For hBNIP-2, translation initiation at uTIS C (ctg) (i.e., the uTIS reported by ribosome profiling in Hek293t cells [32]) resulted in the production of an N-terminally extended BNIP-2 variant. Mutation of the most upstream putative TIS reported, uTIS A, however did not alter the precursor pattern observed in the human and mouse BNIP-2 control setups. Finally, mutation of the database annotated initiator methionine in human and mouse BNIP-2 resulted in the disappearance of the lowest molecular weight band (Additional file 7: Figure S5). Overall, these analyses unequivocally point to the use of alternative translation initiation start sites in the $5^{\prime}$ leader of BNIP-2 leading to the production of N-terminally extended BNIP-2 variants.

\section{Discussion}

Our current and previous degradome analyses led to the identification of human BNIP-2, a pro-apoptotic Bcl-2 family member, as an efficient human and mouse granzyme B substrate. For mGrB however, this represents an unanticipated cleavage event given that neither human nor mouse Bid are cleaved by $\mathrm{mGrB}$ whereas hBNIP-2 is cleaved after the hGrB specific P4-P1 Bid tetrapeptide motif IEAD. Up till now the P4-P1 specificity profile was considered to be the main determinant for substrate recognition. In this study, hBNIP-2 was found to be efficiently processed by both orthologous granzymes $B$ at $\mathrm{IEAD}_{28}$. Interestingly, at the peptide-level $\mathrm{mGrB}$ was found to cleave the hBNIP-2 specific peptide 5-fold more efficiently as compared to hGrB. On the other 


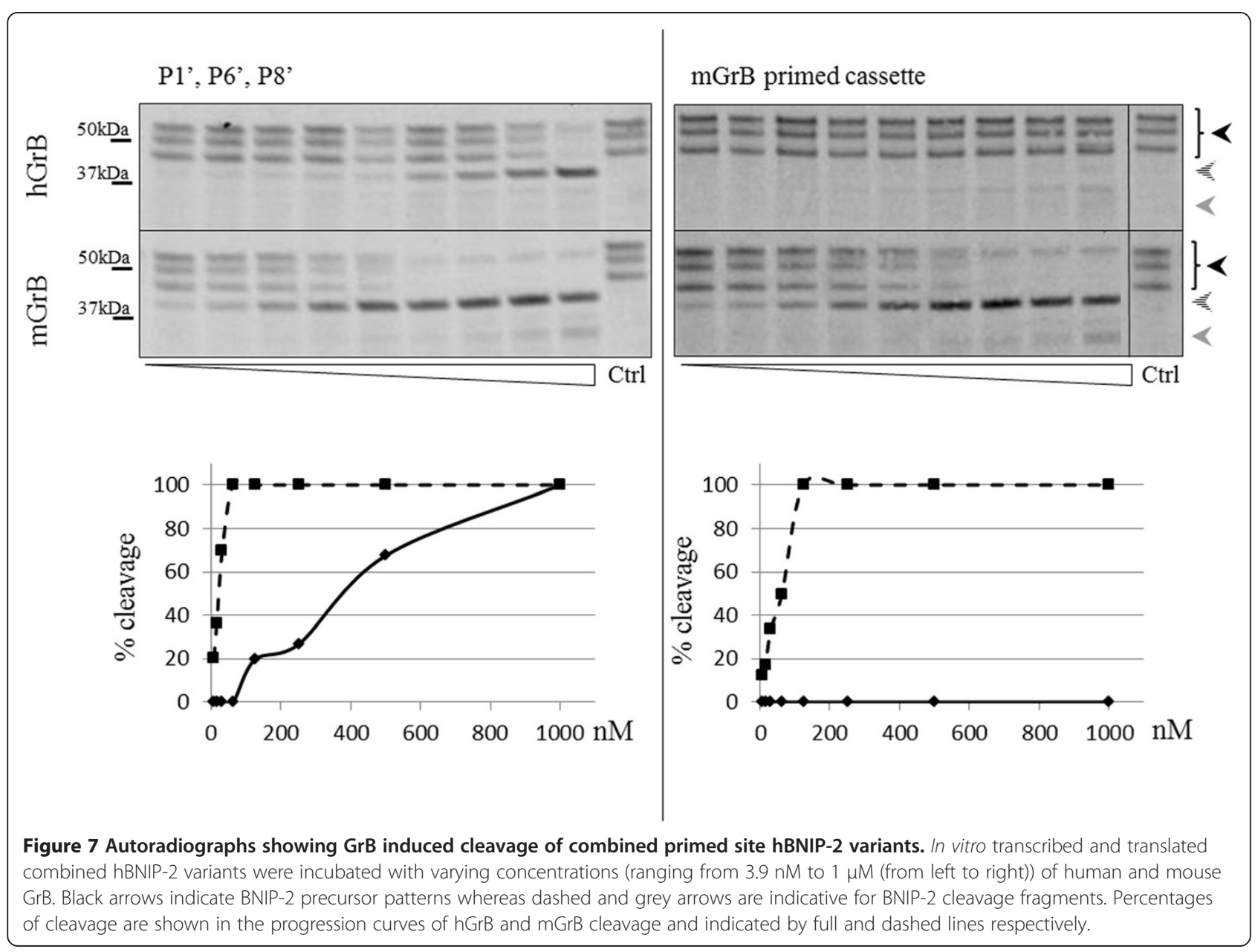

hGrB
mGrB




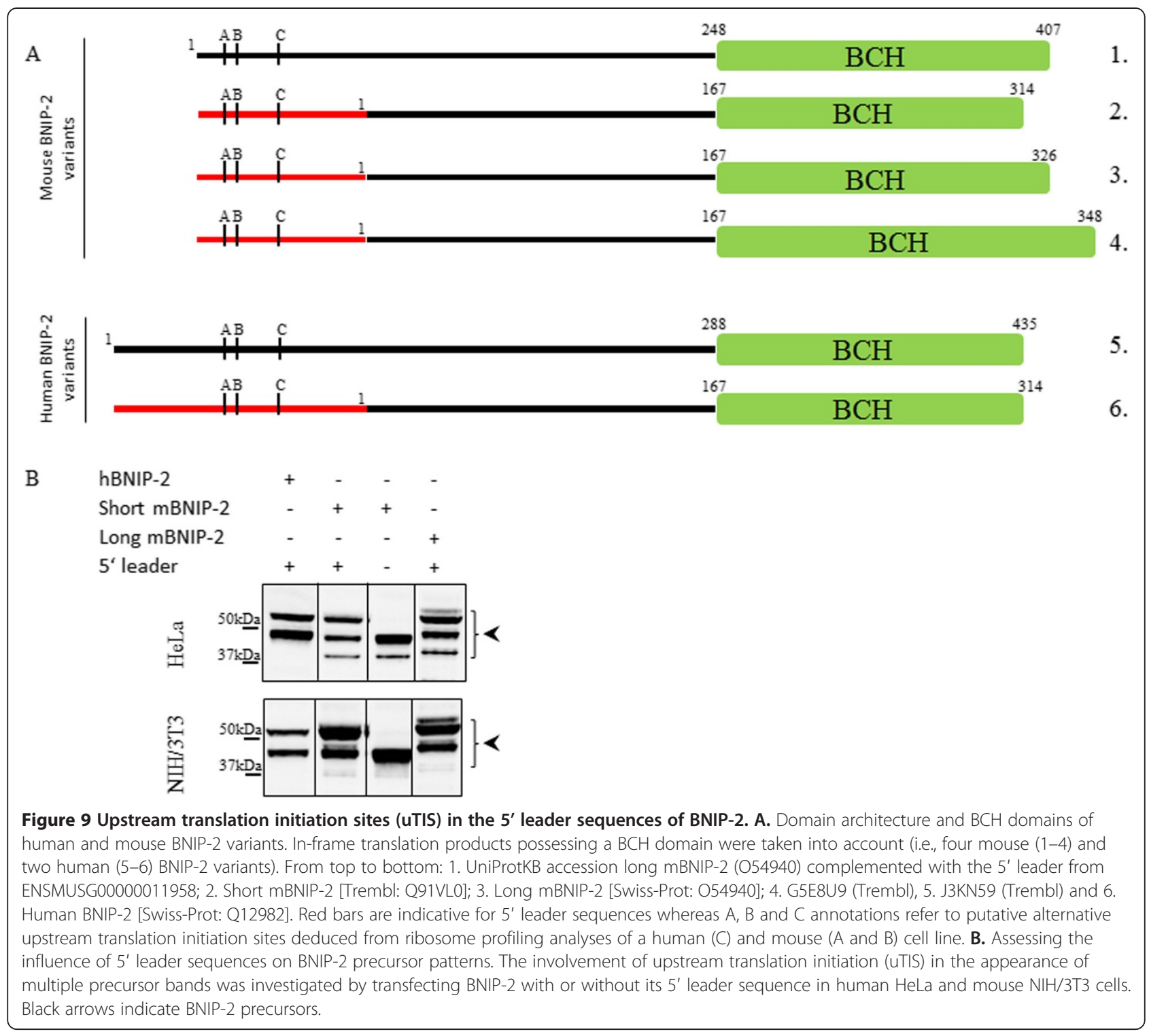

hand, $h \mathrm{GrB}$ appeared to process hBNIP-2 in cell-free extracts more efficiently while $\mathrm{mGrB}$ processing of in vitro translated human and mouse BNIP-2 exceeded hGrB cleavage efficiencies over 25 -fold. These discrepancies in cleavage efficiencies are likely explained by structurally different accessibilities of the cleavage site.

Structural analyses using IUPred [37], SEG [38] and DisoPred [39], all predict the GrB targeted $\mathrm{IEAD}_{28}$ site to reside in an unstructured/disordered region of the human and mouse BNIP-2 orthologs, a feature reminiscent to the $\mathrm{Bid} \mathrm{IEAD}_{75}$ motif residing in an unstructured/disordered region of the available crystal and NMR structures of Bid [40]. Strengthened by the observation that all N-terminal extended BNIP-2 variants display equal GrB cleavage susceptibilities, this implies that, if the overall accessibility of the cleavage site is similar, binding differences and thus differences in the $\mathrm{mGrB}$ and $\mathrm{hGrB}$ cleavage efficiencies must be found in the detail of the extended substrate motif recognized. Remarkably, the most noteworthy sequence context differences between the highly similar human and mouse BNIP-2 sequences were found immediately $\mathrm{C}$-terminal to the scissile bond and more specifically from $\mathrm{P}^{\prime}$ to $\mathrm{P}^{\prime}$. While structural modelling and molecular dynamics simulation cannot reliably model the flexible $\mathrm{N}$-terminus providing further molecular insight, murinization of hBNIP-2 was done to investigate the contribution of each differing amino acid to the higher cleavage efficiency observed for mGrB. Our data show the P3' Ala to Asp mutation as the most critical determinant for the observed differences in $\mathrm{mGrB} / \mathrm{hGrB}$ mediated mBNIP-2 cleavage. On the other hand, next to amino acids present in the P6' and P8' 


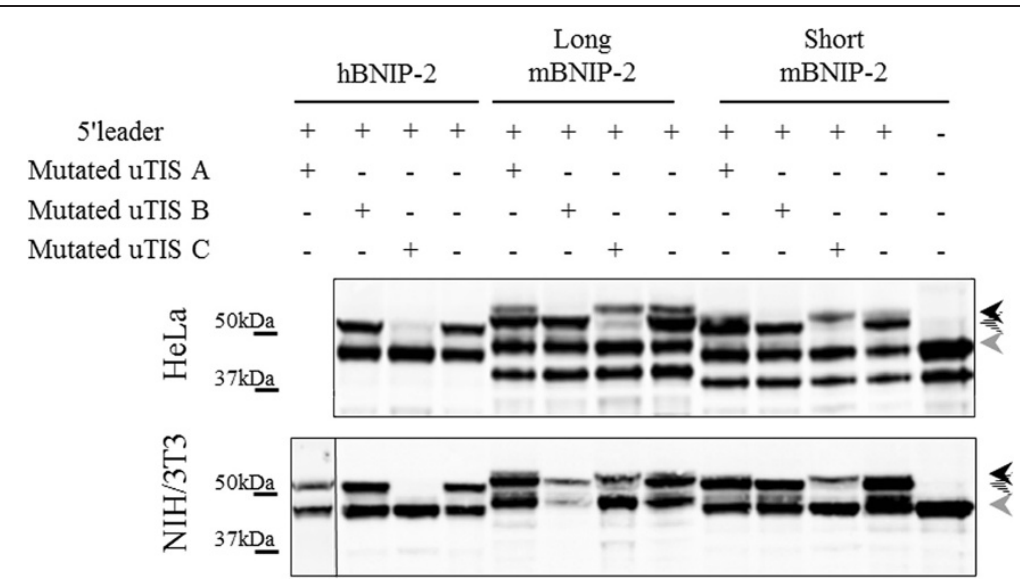

Figure 10 Assessing the influence of 5 ' leader sequences on BNIP-2 precursor patterns. The contribution of alternative translation initiation to the multiple precursor bands was assessed by transfecting HeLa and NIH/3T3 cells with various UTIS mutated BNIP-2 expressing constructs. Whereas mutation of UTIS A did not alter the precursor pattern observed in the human and mouse BNIP-2 control setups, mutation of uTIS B (black arrow) resulted in the disappearance of the precursor band with the highest MW in murine BNIP-2 variants. The dashed arrow is indicative for the N-terminally extended BNIP-2 variant raised upon translation initiation at UTIS C. The BNIP-2 Swiss-Prot database annotated precursor band is annotated with a grey arrow (cfr. Additional file 7: Figure S5).

position, the largest increase in cleavage efficiency was obtained when mutating Ile in the P1' position to Thr. These results are in accordance with the previously reported extended substrate specificity profiles of human and mouse $\mathrm{GrB}$ [1], where a preference for small residues was found at the $\mathrm{P}^{\prime}$ position next to a preference for acidic residues from the P3' position onwards, thus validating the increased cleavage susceptibility of $\mathrm{mGrB}$ and hGrB observed when mutating amino acids in the $\mathrm{P}^{\prime}$ and $\mathrm{P}^{\prime}$ positions to threonine and aspartic acid respectively. Combination of primed site mutations increased $\mathrm{mGrB} / \mathrm{hGrB}$ cleavage ratios over 10 -fold (as compared to wild type hBNIP-2 cleavage; i.e., $\mathrm{P}^{\prime}{ }^{\prime}, \mathrm{P}^{\prime}$ ' and P8' residues) hinting to primed site substrate cooperativity steering GrB cleavage. Further, substitution of all differing hGrB primed site residues following $\mathrm{Asp}_{28}$ by the entire murine primed cassette resulted in cleavage efficiencies approaching those observed for $\mathrm{mGrB}$ cleavage of mBNIP-2. Furthermore, BNIP-2 cleavage in a cellular context was shown to occur upon SLO-mediated GrB delivery, hinting to its physiological relevance.

Comparing kinetic degradomics data of human and mouse GrB obtained in an identical proteome background showed that among the efficiently targeted GrB cleavage sites identified, four were shared between $\mathrm{mGrB}$ (Additional file 1: Table S1) and hGrB [8]. While three out of four were found processed with similar efficiencies, human granzyme B cleavage of caspase-7 was found to proceed less efficiently. More specifically, the neo-N-terminus generated upon granzyme B cleavage ( $\mathrm{S}_{199}$ GPINDTDANPR) carries a glycine residue at the P2' position which was previously shown by us and others $[3,8]$ to be preferred by $\mathrm{mGrB}$, and thus explains the higher efficiency of $\mathrm{mGrB} / \mathrm{hGrB}$ cleavage observed by means of kinetic degradome analyses [8]. An observation in line with the assumption that $\mathrm{mGrB}$ induced apoptosis mainly proceeds via the activation of caspases [2,14].

Interestingly, multiple BNIP-2 precursor bands were observed for endogenous and in vitro translated or overexpressed BNIP-2 in HeLa and NIH/3T3 cells. The omnipresence of alternative translation initiation [41] and the recent finding of potential uTIS within the $5^{\prime}$ leader sequence of BNIP-2 [31,32], led us to investigate whether translation at the reported uTIS were causative for the composite BNIP-2 precursor patterns observed. Transfection of an eukaryotic mBNIP-2 expression vector depleted of the $5^{\prime}$ leader sequence indeed confirmed the usage of at least one uTIS leading to the creation of an N-terminally extended form of the database annotated BNIP-2 sequence, an observation further validated when overexpressing various human and mouse BNIP-2 uTIS mutants in HeLa and NIH/3T3 cells. In the current study, translation initiation at uTIS A, the most upstream uTIS determined by ribosome profiling in mice, could not be observed. This difference could well be explained by the fact that the original BNIP-2 expression constructs only hold part of the $5^{\prime}$ leader sequences, thus potentially lacking regulatory sequences promoting translation initiation at this site [42]. The potential importance of amino terminal BNIP-2 extensions can be exemplified by the observation that an $\mathrm{N}$-terminally extended Bid splice variant has an altered subcellular localization potentially influencing cellular apoptosis [43]. Strikingly, we also observed a cell context dependency of TIS usage, the regulation of which requires follow-up studies. Next, 
Scott et al. reported that both the full-length as well as the GrB generated BNIP-2 fragments induce PARP-1 cleavage upon transfection in HeLa cells [11]. Of note however, expression of (t)BNIP-2 resulted only in a very low percentage of PARP-1 cleavage [11]. Moreover, a $24 \mathrm{~h}$ transfection of the empty vector or a mock control transfection clearly also resulted in PARP-1 cleavage which approximated (t)BNIP-2 induced PARP-1 cleavage. In this study, none of the techniques suitable for the detection and/or quantification of the degree of apoptosis applied, were capable of detecting evidence of cell death in BNIP-2 or tBNIP-2 transfected human (HeLa) and murine (NIH/3T3) cells while positive controls (staurosporin to induce apoptosis) did result in an apoptotic readout, questioning the (cell-type dependent) role of BNIP-2 in apoptotic signaling.

\section{Conclusions}

The differential cleavage susceptibilities of BNIP-2 by orthologous granzymes B can be explained by the presence of different primed site residues in the mouse and human BNIP-2 orthologs, in accordance with differential subsite requirements observed for both granzymes [1-4]. Whether mBNIP-2 plays a pivotal role in $\mathrm{mGrB}$ induced cell death and how alternative translation initiation of BNIP-2 affects BNIP-2 function remains to be elucidated.

\section{Availability of supporting data}

The data set supporting the results of this article is included within the article (and its additional file(s)).

\section{Additional files}

Additional file 1: Table S1. Classification of $37 \mathrm{mGrB}$ generated neo-N-termini and their corresponding substrates. The UniProtKB/Swiss-Prot database primary accession number, protein description, P10-P10' sequence and fold change of the $\mathrm{mGrB}$ generated neo-N-termini are given between 10 and $30 \mathrm{~min}$ and between 30 and $60 \mathrm{~min}$. For each category, proteins are ranked alphabetically according to their protein description. Additional information on the maximum score, identity threshold, isoforms and whether these identical cleavage sites were previously found in the $\mathrm{N}$-terminal COFRADIC analyses screening for efficient hGrB cleavage sites/substrates [8] and analyses on $\mathrm{hGrB}, \mathrm{mGrB}$ and $\mathrm{mGrC}$ respectively are listed [5].

Additional file 2: Table S2. List of primers used to design mutants and attB PCR flanked products. Mutated nucleotides are underlined.

Additional file 3: Figure S1. Autoradiographs showing the resistance of human and mouse BNIP-2 IEAA 28 mutants to GrB induced cleavage. In vitro transcribed and translated human and mouse BNIP-2 IEAA 28 mutants were incubated with varying concentrations (ranging from $3.9 \mathrm{nM}$ to 500 nM (from right to left)) of human or mouse GrB. Black arrows indicate BNIP-2 precursor patterns.

Additional file 4: Figure S2. Autoradiographs showing GrB induced cleavage of murinized human BNIP-2 variants. P4', P6', P8' and P9' differing primed site residues following the identified P4-P1 cleavage site IEAD in hBNIP-2 were mutated to their corresponding mBNIP-2 amino acids. In vitro transcribed and translated BNIP-2 variants were incubated with varying concentrations (ranging from $1.95 \mathrm{nM}$ to $1 \mu \mathrm{M}$ (from left to right)) of human or mouse granzyme B. Black arrows indicate BNIP-2 precursor patterns, whereas dashed and grey arrows are indicative for BNIP-2 cleavage fragments. Percentages of cleavage are shown in the progression curves of $\mathrm{hGrB}$ and $\mathrm{mGrB}$ cleavage and indicated by full and dashed lines respectively.

Additional file 5: Figure S3. ClustalW multiple sequence alignment of human and mouse BNIP-2 variants. The amino acid sequences of the longest hBNIP-2 annotated protein (annotated as hBNIP-2; UniProtKB accession [Trembl: J3KN59]) as well as the N-terminally extended UTIS variants of hBNIP2 [Swiss-Prot: Q12982] were used in the alignment. The longest mBNIP-2 variant is based on 054940 (Swiss-Prot) complemented with the $5^{\prime}$ leader from ENSMUSG00000011958 whereas other variants of mBNIP-2 (short mouse BNIP-2 [Trembl: Q91VL0] and long mBNIP-2

[Swiss-Prot: O54940]) are depicted together with their postulated N-terminal extensions. The black box highlights the P4-P1 IEAD recognition motif whereas the red box assigns the database annotated initiator Met of mouse and human BNIP-2 proteins.

Additional file 6: Figure S4. Cloned sequences of human (A), short mouse (B) and long mouse (C) BNIP-2. Alternative translation initiation sites are indicated in italics. Red and green letters denote the putative upstream translation initiation sites identified by means of ribosome profiling in mouse and human cells respectively. Below each nucleotide sequence, amino acid sequences of putative translation products are given.

Additional file 7: Figure S5. Assessing the influence of $5^{\prime}$ leader sequences on BNIP-2 precursor patterns. Assignment of the precursor band corresponding to the database annotated protein (human BNIP-2:[Swiss-Prot: Q12982], short mouse BNIP-2:[Trembl: Q91VL0] and long mouse BNIP-2: [Swiss-Prot: O54940]) by mutating their respective initiator methionines. A grey arrow points to the UniProtKB annotated BNIP-2 variants, while black and dashed arrows indicate the BNIP-2 variants associated with translation initiation at UTIS B and UTIS C respectively.

\section{Abbreviations}

(h/m)GrB: (human/mouse) granzyme B; (t)Bid: (truncated) BH3-interacting domain death agonist; ACD3: Trideutero-acetyl; aTIS: Alternative translation initiation site; $\mathrm{BCH}$ : BNIP-2 and Cdc42GAP homology; $\mathrm{BH}$ : BCl-2 homology; BNIP-2: BCL2/adenovirus E1B $19 \mathrm{kDa}$ protein-interacting protein 2; COFRADIC: Combined fractional Diagonal chromatography; dbTIS: Database annotated translation initiation site; f.c.: Final concentration; IAA: Iodoacetamide; IAP: Inhibitor of apoptosis; ORF: Open reading frame; PARP-1: Poly (ADP) ribose polymerase 1; SCX: Strong cation exchange; SILAC: Stable isotope labeling by amino acids in cell culture; TNBS: 2,4,6-trinitrobenzenesulfonic acid; UTIS: Upstream translation initiation site; UTR: Untranslated region; zVAD-fmk: $\mathrm{N}$-Benzyloxycarbonyl-Val-Ala-Asp (O-methyl) fluoromethyl ketone.

\section{Competing interests}

The authors declare that they have no competing interests.

\section{Authors' contributions}

PVD and KP contributed equally to this work. PVD and KP designed research; PVD, KP, GV and VJ performed research; PVD, KP and SMS analyzed data; PVD and KP wrote the paper; PVD and KG supervised research. All authors read and approved the final manuscript.

\section{Acknowledgements}

We thank Kim Van Der Eecken for technical assistance. We also thank Prof. P. Bird from the Department of Biochemistry and Molecular Biology, Monash University, Victoria 3800, Australia for providing human and mouse granzyme B. P.V.D. is a Postdoctoral Fellow of the Research Foundation - Flanders (FWO-Vlaanderen). K.P. was supported by a PhD grant from the Institute for the Promotion of Innovation through Science and Technology in Flanders (IWT-Vlaanderen). P.V.D acknowledges support from the Research Foundation Flanders (FWO-Vlaanderen), project number G.0269.13 N.

\section{Author details}

'Department of Medical Protein Research, VIB, Flanders Interuniversity Institute for Biotechnology, Ghent University, A. Baertsoenkaai 3, B9000 
Ghent, Belgium. ${ }^{2}$ Department of Biochemistry, Ghent University, B-9000 Ghent, Belgium. ${ }^{3}$ Bioinformatics Institute (BII), Agency for Science, Technology and Research (A*STAR), Singapore 138671, Singapore. ${ }^{4}$ School of Biological Sciences (SBS), Nanyang Technological University (NTU), Singapore 637551, Singapore.

Received: 9 May 2014 Accepted: 13 August 2014

Published: 10 September 2014

\section{References}

1. Van Damme P, Maurer-Stroh S, Plasman K, Van Durme J, Colaert N, Timmerman E, De Bock PJ, Goethals M, Rousseau F, Schymkowitz J, Vandekerckhove J, Gevaert K: Analysis of protein processing by N-terminal proteomics reveals novel species-specific substrate determinants of granzyme B orthologs. Mol Cell Proteomics 2009, 8(2):258-272.

2. Cullen SP, Adrain C, Luthi AU, Duriez PJ, Martin SJ: Human and murine granzyme B exhibit divergent substrate preferences. J Cell Biol 2007, 176(4):435-444.

3. Kaiserman D, Bird CH, Sun J, Matthews A, Ung K, Whisstock JC, Thompson PE, Trapani JA, Bird PI: The major human and mouse granzymes are structurally and functionally divergent. J Cell Biol 2006, 175(4):619-630.

4. Casciola-Rosen L, Garcia-Calvo M, Bull HG, Becker JW, Hines T, Thornberry $N A$, Rosen $A$ : Mouse and human granzyme $B$ have distinct tetrapeptide specificities and abilities to recruit the bid pathway. J Biol Chem 2007, 282(7):4545-4552.

5. Plasman K, Maurer-Stroh S, Gevaert K, Van Damme P: Holistic view on the extended substrate specificities of orthologous granzymes. J Proteome Res 2014, 13(4):1785-1793.

6. Schechter I, Berger A: On the size of the active site in proteases. I. Papain Biochem Biophys Res Commun 1967, 27(2):157-162.

7. Agard NJ, Mahrus S, Trinidad JC, Lynn A, Burlingame AL, Wells JA: Global kinetic analysis of proteolysis via quantitative targeted proteomics. Proc Natl Acad Sci U S A 2012, 109(6):1913-1918.

8. Plasman K, Van Damme P, Kaiserman D, Impens F, Demeyer K, Helsens K, Goethals M, Bird PI, Vandekerckhove J, Gevaert K: Probing the efficiency of proteolytic events by positional proteomics. Mol Cell Proteomics 2011, 10(2):M110 003301.

9. Schlage P, Egli FE, Nanni P, Wang LW, Kizhakkedathu JN, Apte SS, auf dem Keller U: Time-resolved analysis of the matrix metalloproteinase 10 substrate degradome. Mol Cell Proteomics 2014, 13(2):580-593.

10. Trapani JA, Sutton VR: Granzyme B: pro-apoptotic, antiviral and antitumor functions. Curr Opin Immunol 2003, 15(5):533-543.

11. Scott GB, Bowles PA, Wilson EB, Meade JL, Low BC, Davison A, Blair GE, Cook GP: Identification of the BCL2/adenovirus E1B-19 K protein-interacting protein 2 (BNIP-2) as a granzyme B target during human natural killer cell-mediated killing. Biochem J 2010, 431(3):423-431.

12. Waterhouse NJ, Sedelies KA, Browne KA, Wowk ME, Newbold A, Sutton VR, Clarke CJ, Oliaro J, Lindemann RK, Bird PI, Johnstone RW, Trapani JA: A central role for Bid in granzyme B-induced apoptosis. J Biol Chem 2005, 280(6):4476-4482.

13. Waterhouse NJ, Sedelies KA, Trapani JA: Role of Bid-induced mitochondrial outer membrane permeabilization in granzyme B-induced apoptosis. Immunol Cell Biol 2006, 84(1):72-78.

14. Thomas DA, Scorrano L, Putcha GV, Korsmeyer SJ, Ley TJ: Granzyme B can cause mitochondrial depolarization and cell death in the absence of BID, BAX, and BAK. Proc Natl Acad Sci U S A 2001, 98(26):14985-14990.

15. Goping IS, Barry M, Liston P, Sawchuk T, Constantinescu G, Michalak KM, Shostak I, Roberts DL, Hunter AM, Korneluk R, Bleackley RC: Granzyme B-induced apoptosis requires both direct caspase activation and relief of caspase inhibition. Immunity 2003, 18(3):355-365.

16. Boyd JM, Malstrom S, Subramanian T, Venkatesh LK, Schaeper U, Elangovan B, D'Sa-Eipper C, Chinnadurai G: Adenovirus E1B 19 kDa and Bcl-2 proteins interact with a common set of cellular proteins. Cell 1994, 79(2):341-351.

17. Zhou YT, Guy GR, Low BC: BNIP-2 induces cell elongation and membrane protrusions by interacting with $\mathrm{Cdc} 42$ via a unique Cdc42-binding motif within its BNIP-2 and Cdc42GAP homology domain. Exp Cell Res 2005, 303(2):263-274.

18. Kang JS, Bae GU, Yi MJ, Yang YJ, Oh JE, Takaesu G, Zhou YT, Low BC, Krauss RS: A Cdo-Bnip-2-Cdc42 signaling pathway regulates $\mathrm{p} 38 \mathrm{alpha/beta}$ MAPK activity and myogenic differentiation. J Cell Biol 2008, 182(3):497-507.
19. Pan CQ, Low BC: Functional plasticity of the BNIP-2 and Cdc42GAP Homology $(\mathrm{BCH})$ domain in cell signaling and cell dynamics. FEBS Lett 2012, 586(17):2674-2691

20. Belcredito S, Vegeto E, Brusadelli A, Ghisletti S, Mussi P, Ciana P, Maggi A: Estrogen neuroprotection: the involvement of the $\mathrm{BCl}-2$ binding protein BNIP2. Brain Res Brain Res Rev 2001, 37(1-3):335-342.

21. Valencia CA, Cotten SW, Liu R: Cleavage of BNIP-2 and BNIP-XL by caspases. Biochem Biophys Res Commun 2007, 364(3):495-501.

22. Ju W, Valencia CA, Pang H, Ke Y, Gao W, Dong B, Liu R: Proteome-wide identification of family member-specific natural substrate repertoire of caspases. Proc Natl Acad Sci U S A 2007, 104(36):14294-14299.

23. Sall A, Zhang HM, Qiu D, Liu Z, Yuan J, Lim T, Ye X, Marchant D, McManus B, Yang D: Pro-apoptotic activity of mBNIP-21 depends on its BNIP-2 and Cdc42GAP homology (BCH) domain and is enhanced by coxsackievirus B3 infection. Cell Microbiol 2010, 12(5):599-614.

24. Zhang HM, Yanagawa B, Cheung P, Luo H, Yuan J, Chau D, Wang A, Bohunek L, Wilson JE, McManus BM, Yang D: Nip21 gene expression reduces coxsackievirus $B 3$ replication by promoting apoptotic cell death via a mitochondria-dependent pathway. Circ Res 2002, 90(12):1251-1258.

25. Staes A, Impens F, Van Damme P, Ruttens B, Goethals M, Demol H, Timmerman E, Vandekerckhove J, Gevaert K: Selecting protein N-terminal peptides by combined fractional diagonal chromatography. Nat Protoc 2011, 6(8):1130-1141.

26. Ghesquiere B, Van Damme J, Martens L, Vandekerckhove J, Gevaert K: Proteome-wide characterization of $\mathrm{N}$-glycosylation events by diagonal chromatography. J Proteome Res 2006, 5(9):2438-2447.

27. Staes A, Van Damme P, Helsens K, Demol H, Vandekerckhove J, Gevaert K: Improved recovery of proteome-informative, protein $\mathrm{N}$-terminal peptides by combined fractional diagonal chromatography (COFRADIC). Proteomics 2008, 8(7):1362-1370.

28. Vizcaino JA, Cote RG, Csordas A, Dianes JA, Fabregat A, Foster JM, Griss J, Alpi E, Birim M, Contell J, O'Kelly G, Schoenegger A, Ovelleiro D, Perez-Riverol Y, Reisinger F, Rios D, Wang R, Hermjakob H: The PRoteomics IDEntifications (PRIDE) database and associated tools: status in 2013. Nucleic Acids Res 2013, 41(Database issue):D1063-D1069.

29. Zapata JM, Takahashi R, Salvesen GS, Reed JC: Granzyme release and caspase activation in activated human T-lymphocytes. J Biol Chem 1998, 273(12):6916-6920.

30. Demon D, Van Damme P, Vanden Berghe T, Deceuninck A, Van Durme J, Verspurten J, Helsens K, Impens F, Wejda M, Schymkowitz J, Rousseau F, Madder A, Vandekerckhove J, Declercq W, Gevaert K, Vandenabeele P: Proteome-wide substrate analysis indicates substrate exclusion as a mechanism to generate caspase- 7 versus caspase-3 specificity. Mol Cell Proteomics 2009, 8(12):2700-2714.

31. Ingolia NT, Lareau LF, Weissman JS: Ribosome profiling of mouse embryonic stem cells reveals the complexity and dynamics of mammalian proteomes. Cell 2011, 147(4):789-802.

32. Lee S, Liu B, Huang SX, Shen B, Qian SB: Global mapping of translation initiation sites in mammalian cells at single-nucleotide resolution. Proc Natl Acad Sci U S A 2012, 109(37):E2424-E2432.

33. Ong SE, Blagoev B, Kratchmarova I, Kristensen DB, Steen H, Pandey A, Mann $\mathrm{M}$ : Stable isotope labeling by amino acids in cell culture, SILAC, as a simple and accurate approach to expression proteomics. Mol Cell Proteomics 2002, 1(5):376-386

34. Menschaert G, Van Criekinge W, Notelaers T, Koch A, Crappe J, Gevaert K, Van Damme P: Deep Proteome Coverage Based on Ribosome Profiling Aids Mass Spectrometry-based Protein and Peptide Discovery and Provides Evidence of Alternative Translation Products and Near-cognate Translation Initiation Events. Mol Cell Proteomics 2013, 12(7):1780-1790.

35. Low BC, Lim YP, Lim J, Wong ES, Guy GR: Tyrosine phosphorylation of the $\mathrm{BCl}$-2-associated protein BNIP-2 by fibroblast growth factor receptor-1 prevents its binding to Cdc42GAP and Cdc42. J Biol Chem 1999, 274(46):33123-33130.

36. Qin W, Hu J, Guo M, Xu J, Li J, Yao G, Zhou X, Jiang H, Zhang P, Shen L, Wan D, Gu J: BNIPL-2, a novel homologue of BNIP-2, interacts with BCl-2 and Cdc42GAP in apoptosis. Biochem Biophys Res Commun 2003, 308(2):379-385.

37. Dosztanyi Z, Csizmok V, Tompa P, Simon I: IUPred: web server for the prediction of intrinsically unstructured regions of proteins based on estimated energy content. Bioinformatics 2005, 21(16):3433-3434 
38. Wootton JC, Federhen S: Analysis of compositionally biased regions in sequence databases. Methods Enzymol 1996, 266:554-571.

39. Ward JJ, Sodhi JS, McGuffin L, Buxton BF, Jones DT: Prediction and functional analysis of native disorder in proteins from the three kingdoms of life. J Mol Biol 2004, 337(3):635-645.

40. Chou JJ, Li H, Salvesen GS, Yuan J, Wagner G: Solution structure of BID, an intracellular amplifier of apoptotic signaling. Cell 1999, 96(5):615-624.

41. Van Damme P, Gawron D, Van Criekinge W, Menschaert G: N-terminal proteomics and ribosome profiling provide a comprehensive view of the alternative translation initiation landscape in mice and men. Mol Cell Proteomics 2014, 13:1245-1261.

42. Barrett LW, Fletcher S, Wilton SD: Regulation of eukaryotic gene expression by the untranslated gene regions and other non-coding elements. Cell Mol Life Sci 2012, 69(21):3613-3634.

43. Renshaw SA, Dempsey CE, Barnes FA, Bagstaff SM, Dower SK, Bingle CD, Whyte MK: Three novel Bid proteins generated by alternative splicing of the human Bid gene. J Biol Chem 2004, 279(4):2846-2855.

doi:10.1186/1471-2091-15-21

Cite this article as: Van Damme et al:: Importance of extended protease substrate recognition motifs in steering BNIP-2 cleavage by human and mouse granzymes B. BMC Biochemistry 2014 15:21.

\section{Submit your next manuscript to BioMed Central and take full advantage of:}

- Convenient online submission

- Thorough peer review

- No space constraints or color figure charges

- Immediate publication on acceptance

- Inclusion in PubMed, CAS, Scopus and Google Scholar

- Research which is freely available for redistribution 